\title{
An LC-MS/MS Method for Determination of Imperatorin and Isoimperatorin in Rat Plasma and Application to a Pharmacokinetic Study
}

\author{
Junbo Xing, Hong Cao, Yumin Chen, Caihong Shui, Tingting Shan, Dan Hu \\ Department of Traditional Chinese Medicine, Institute for Drug and Instrument Control of Health Department, GLD of PLA, Beijing, \\ China. \\ Email: junboxing89@sina.com
}

Received June $19^{\text {th }}, 2013$; revised July $21^{\text {st }}, 2013$; accepted August $1^{\text {st }}, 2013$

Copyright (C) 2013 Junbo Xing et al. This is an open access article distributed under the Creative Commons Attribution License, which permits unrestricted use, distribution, and reproduction in any medium, provided the original work is properly cited.

\begin{abstract}
A highly sensitive liquid chromatography-tandem mass spectrometric (LC-MS/MS) method was developed for the determination of imperatorin and isoimperatorin in rat plasma using psoralen as internal standard. Analyses were performed on a Shimadzu HPLC system using a Shimadzu C18 column eluted with a mobile phase of methanol-water (25:75), containing $0.1 \%$ formic acid at a flow rate of $0.4 \mathrm{ml} / \mathrm{min}$. The detection was performed by positive ion electrospray ionization in multiple reactions monitoring mode, monitoring the transitions $\mathrm{m} / \mathrm{z} 203 \rightarrow 147$ and $\mathrm{m} / \mathrm{z} 203 \rightarrow 159$ for imperatorin and isoimperatorin, respectively. The method was validated over the concentration range of $2.5-1000.0 \mathrm{ng} / \mathrm{mL}$ for imperatorin and isoimperatorin. The limits of detection and quantification were 1.0 and $3.0 \mathrm{ng} / \mathrm{mL}$, repectively for both analytes. The intra-day and inter-day accuracy and precision of the assay were less than $10.9 \%$. This method has been applied successfully to a pharmacokinetic study involving the intragastric administration of extract of Angelicae Dahuricae Radix to rats.
\end{abstract}

Keywords: Imperatorin; Isoimperatorin; LC-MS/MS; Pharmacokinetic

\section{Introduction}

Angelicae Dahuricae Radix (Chinese herbal name: BaiZhi), the dried radix of Angelica dahurica (Fisch. ex hoffm.) Benth. Et Hook. f. and A. dahurica (Fisch. ex hoffm.) Benth. et Hook. f. var. formosana (Boiss.) Shan et Yuan, is a well-known Chinese herbal medicine which was originally described in Shennong Materia Medica, the earliest Pharmacopoeia of China in Eastern Han [1,2]. It has been widely used in China for over 2000 years for the treatment of headache, toothache, nose congestion resulting from cold and the reduction of swelling and pain from sores and wounds [3,4], and now it also has been reported as having antioxidative activity against 2 , 2'-azobis (2-aminodinopropane) dihydrochloride (AAPH) induced cellular damage [5,6], chronic toxicology [7], anti-inflammatory, anti-febrile, anti-tumor, analgesic and antipyretic activity [8-13], and treating migrain [14]. The coumarins imperatorin and isoimperatorin (structures shown in Figure 1) are the major active components of Angelicae Dahuricae Radix.
To date, there have already been some preliminary researches about the quantitative analysis of coumarins in different plants and plant products, for example, determination of imperatorin in rat plasma by HPLC [15], simultaneous determination of byak-angelicin and oxypeucedanin hydrate in rat plasma by HPLC-UV [16], determination and pharmacokinetic study of oxypeucedanin hydrate and byak-angelicin after oral administration of Angelica dahurica extracts in mongrel dog plasma by HPLC [17], determination of ochratoxin A and ochratoxin B in traditional Chinese medicines by HPLCtandem mass spectrometry [18], characterization of coumarins in Radix Glehniae by liquid chromatography coupled with triple quadrupole-linear ion trap mass spectrometry [19], analysis of isoimperatorin in rat plasma, and extraction products from Angelica dahurica (Fisch. ex Hoffm.) Benth. et Hook by GC-MS [20-23], simultaneous determination of liquiritin, glycyrrhizin, hesperidin, cinnamic acid, cinnamaldehyde, honokiol and magnolol in the traditional Chinese medicinal preparation $\mathrm{Wu}$ Ji-San by high-performance liquid chromatography [24], 


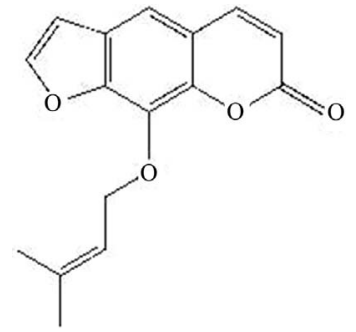

Imperatorin, (MW:270)<smiles>O=c1ccc2cc3ccoc3cc2o1</smiles>

Figure 1. Chemical structures of imperatorin, isoimperatorin and internal standard psoralen.

simultaneous characterization and quantitation of 11 coumarins in Radix Angelicae Dahuricae by HPLC-ESI-MS/ MS [25], HPLC Fingerprint of Processed Radix Angelicae Dahuricae [26-28], molecular identification-anchored primer amplification polymorphism DNA [29], simultaneous determination of sevel furocoumarins in Angelicae dahuricae radix by HPLC [30-33]. Determination of total content of coumarin in Angelica dahurica by UV spectrophotometry [34].

Despite the extensive literature on the pharmacology of them, little information is available relating to their pharmacokinetics. As a knowledge of the pharmacokinetics can help us explain and predict a variety of events related to the efficacy and toxicity of herbal preparations, it is important to investigate the pharmacokinetics of their active constituents. To the best of our knowledge, there has been no method for simultaneous characterization and quantitation of imperatorin and isoimperatorin in rat plasma after oral administration of Angelicae dahuricae radix extract by HPLC-MS/MS in MRM mode by now. As a result, the objective of the present study was to develop a highly selective LC-MS/ MS method for the quantification of imperatorin and isoimperatorin in rat plasma with a view to understanding the pharmacokinetics and oral bioavailability of imperatorin and isoimperatorin in rats following oral administration of Angelicae dahuricae radix extract.

\section{Experimental}

\subsection{Chemicals and Reagents}

The dried radix of Angelica dahurica (Fisch. ex hoffm.) Benth. Et Hook. $f$. was purchased from Lvye Traditional Chinese Medicine Co. Ltd. (Beijing, China), a voucher specimen (No. ZHYJS 20090201) was deposited in Institute for Drug and Instrument Control of Health Dept.
GLD of PLA for future reference. Imperatorin, isoimperatorin, and psoralen used as internal standard (IS) (structure in Figure 1) were purchased from the National Institute for the Control of Pharmaceutical and Biological Products (Beijing, China). Methanol and formic acid of HPLC grade was obtained from Fisher Co. Ltd. (Emerson, IA, USA). All other chemicals were of analytical grade and purchased from Beijing Chemical Reagent Company (Beijing, China). Milli-Q (Milford, MA, USA) water was used throughout the study. Healthy Wistar rats weighing 180 - $220 \mathrm{~g}$ were obtained from the Experimental Animals Centers of Beijing University of Traditional Chinese Medicine.

\subsection{LC-MS/MS Instrumentation and Analytical Conditions}

An Agilent 6410A triple quadrupole LC-MS system (Agilent Corporation, MA, USA) equipped with G1311A quaternary pump, G1322A vacuum degasser, G1329A autosampler and G1316A therm. Column compartments was used for all analyses. The system was controlled by MassHunter software (Agilent Corporation, MA, USA).

The separation was performed by a Agilent SB-C18 column $(50 \times 2.1 \mathrm{~mm}, 3.5 \mu \mathrm{m}$, Agilent Corporation, MA, USA. PN871700-902) with an isocratic mobile phase consisted of methanol-water-formic acid (25:75:0.1, $\mathrm{v} / \mathrm{v} / \mathrm{v}$ ) at a flow rate of $0.4 \mathrm{~mL} / \mathrm{min}$. The column was maintained at $40^{\circ} \mathrm{C}$ and the injection volume was $2 \mu \mathrm{L}$. High-purity nitrogen was provided by a liquid nitrogen tank.

Ionization was achieved using electrospray in the positive mode with the spray voltage set at $4000 \mathrm{~V}$. Nitrogen was used as nebulizer gas and nebulizer pressure was set at 40 psi with a source temperature of $105^{\circ} \mathrm{C}$. Desolvation gas (nitrogen) was heated to $350^{\circ} \mathrm{C}$ and delivered at a flow rate of $9 \mathrm{~mL} / \mathrm{min}$. For collisioninduced dissociation (CID), high purity nitrogen was used as collision gas at a pressure of $0.1 \mathrm{MPa}$. Quantification was performed using multiple reactions monitoring (MRM) mode at $\mathrm{m} / \mathrm{z} \quad 203 \rightarrow 147$ for imperatorin, $\mathrm{m} / \mathrm{z} \quad 203 \rightarrow 159$ for isoimperatorin and $\mathrm{m} / \mathrm{z} \quad 131 \rightarrow 115$ for psoralen. The fragmentor energies of MS1 for imperatorin, isoimperatorin and psoralen were set at $150 \mathrm{~V}$. The optimized collision energies of 25, 25 and $40 \mathrm{eV}$ were used for imperatorin, isoimperatorin and psoralen, respectively. The peak widths of precursor and product ions were maintained at $0.7 \mathrm{amu}$ at half-height in the MRM mode.

\subsection{Preparation of Calibration Standards and Quality Control Samples}

Stock solutions of imperatorin, isoimperatorin and IS were prepared all in 50\% methanol containing $0.1 \%$ 
formic acid at a concentration of $100 \mu \mathrm{g} / \mathrm{mL}$. The working standard rat's plasma solutions were serially diluted to a concentration of $2.5,5,10,50,100,200,500,750$, $1000 \mathrm{ng} / \mathrm{ml}$ of imperatorin and isoimperatorin with blank rat plasma. An internal standard was prepared in solution aboved at a concentration of $500 \mathrm{ng} / \mathrm{ml}$. Quality control (QC) samples were prepared in the same way as the calibration samples, representing low, middle and high concentrations of imperatorin and isoimperatorin in plasma at $5,100,750 \mathrm{ng} / \mathrm{mL}$.

\subsection{Preparation of Angelicae Dahuricae Radix Extract}

Angelicae Dahuricae Radix (500 g) was refluxed with $95 \%$ ethanol $(5 \mathrm{~L} 2 \times)$ at $100^{\circ} \mathrm{C}$ for $2 \mathrm{~h}$. The extraction solutions were combined, ethanol was removed under reduced pressure. The residue was suspended in water and extracted with petroleum ether to remove lipids. The aqueous phase was then extracted with ethyl acetate and the resulting solution was evaporated vacuum and offered a brown residue. Altogether $38 \mathrm{~g}$ of powder was obtained and the production rate was approximately $7.6 \%$. The residue was dissolved in $0.9 \%$ saline, to give an extract with a concentration of $0.6 \mathrm{~g} / \mathrm{mL}$ (expressed as the weight of raw material of Angelicae dahuricae Radix).

\subsection{Plasma Sample Preparation}

To a $100 \mu \mathrm{L}$ aliquot of plasma sample, $100 \mu \mathrm{l}$ of ethyl acetate were added. Samples were then extracted by vortex shaking for $15.0 \mathrm{~min}$. Following centrifugation at $10,000 \mathrm{rpm}$ for $10 \mathrm{~min}$, then kept at $-20^{\circ} \mathrm{C}$ for $6 \mathrm{~h}$, and the organic layer was transferred to another tube and evaporated at room temperature under a gentle stream of nitrogen. The dry residue was then reconstituted with 200 $\mu 1$ IS solution $(0.5 \mu \mathrm{g} / \mathrm{ml})$ and vortex-mixed for $20 \mathrm{~s}$. A 2 $\mu 1$ solution was injected into the HPLC-MS/ESI system.

\subsection{Drug Administration and Sample Collection}

Animal experiments were carried out according to institutional guidelines for the care and the use of laboratory animals, and approved by the Animal Ethics Committee of Institute for Drug and Instrument Control of Health Dept. GLD of PLA. Eight male Wistar rats (180 - 220 g) were obtained from the laboratory animal center of Academy of Military Medical Sciences. All rats were deprived of food but given free access to water for $12 \mathrm{~h}$ before and during the experiment. Total baizhi extract was dissolved in $0.9 \%$ saline immediately before intragastric administration and the volume was adjusted at 6.0 $\mathrm{g} / \mathrm{kg}$ for rats (expressed as the weight of raw material of Angelicae Dahuricae Radix). A blood sample $(0.1 \mathrm{~mL})$ was collected from the suborbital vein into heparinized tubes at $0,1,2,3,4,5,6,8,10,12,16$ and $24 \mathrm{~h}$ following drug administration. Plasma samples were processed as described in Section 2.5.

\section{Results and Discussions}

\subsection{Chromatographic Separations}

The HPLC-MS/MS in the MRM mode provided a highly selective method for the determination of imperatorin, isoimperatorin and psoralen (I.S.). The analytes were easily protonated and generated positive product ions. Imperatorin was identified at $\mathrm{m} / \mathrm{z} 271.1$ [imperatorin $+\mathrm{H}]^{+}$, $\mathrm{m} / \mathrm{z} 203.1\left[271.1-\mathrm{C}_{5} \mathrm{H}_{8}\right]^{+}$and $\mathrm{m} / \mathrm{z} 147[203.1-2 \mathrm{CO}]^{+}$, isoimperatorin at $\mathrm{m} / \mathrm{z} 271.1$ [isoimperatorin $+\mathrm{H}]^{+}, \mathrm{m} / \mathrm{z}$ $203\left[271.1-\mathrm{C}_{5} \mathrm{H}_{8}\right]^{+}$and $\mathrm{m} / \mathrm{z} 147[203.1-2 \mathrm{CO}]^{+}$, psoralen at $\mathrm{m} / \mathrm{z} 247.1$ [I.S. $+\mathrm{H}]^{+}, \mathrm{m} / \mathrm{z} 131.1[187.1-2 \mathrm{CO}]^{+}$and $\mathrm{m} / \mathrm{z}$ $115.1\left[187.1 .1-\mathrm{CO}_{2}-\mathrm{CO}\right]^{+}$, respectively. The retention time was approximately $0.9,1.5$ and $2.3 \mathrm{~min}$, respectively. Typical chromatograms resulting from the analysis of various plasma samples are shown in Figure 2. No endogenous substance or chemical components were observed to interfere with the drugs and internal standard over the concentration range.

\subsection{Recovery}

We compared three extraction solvents (acetic ether, ether and methyl t-butyl ether) and finally choose ethyl acetate as the solvent for liquid-liquid extraction, because it has an appropriate recovery and good evaporability. The time of extraction and centrifugation was also shortened. The extraction recoveries of the analyte and the IS were evaluated by comparing the peak areas of the analyte and the IS in pre- and post-extraction plasma samples from six different individuals at the corresponding concentrations. The extraction recoveries of the two components at concentrations of $500 \mathrm{ng} / \mathrm{ml}$ for the analyte were $85.2 \% \pm 5.2 \%$ and $88.6 \% \pm 6.5 \%$, respectively, and for the IS was $87.6 \% \pm 5.4 \%$.

\subsection{Calibration Curve}

The standard curve obtained from detection of plasma containing known amounts of the two compounds was linear over the quantities ranges from 2.5 to $1000 \mathrm{ng} / \mathrm{mL}$ in $0.1 \mathrm{~mL}$ rat plasma. The calibration curves were found to be linear and could be described by the regression equations as shown in Table 1 with coefficience of over 0.999 . The lowest limits of quantitation for two substances were $2.5 \mathrm{ng} / \mathrm{mL}$ in $0.1 \mathrm{~mL}$ rat plasma. This sensitivity has proven useful in the analysis of pharmacokinetic data of rats treated after intragastric administration. 

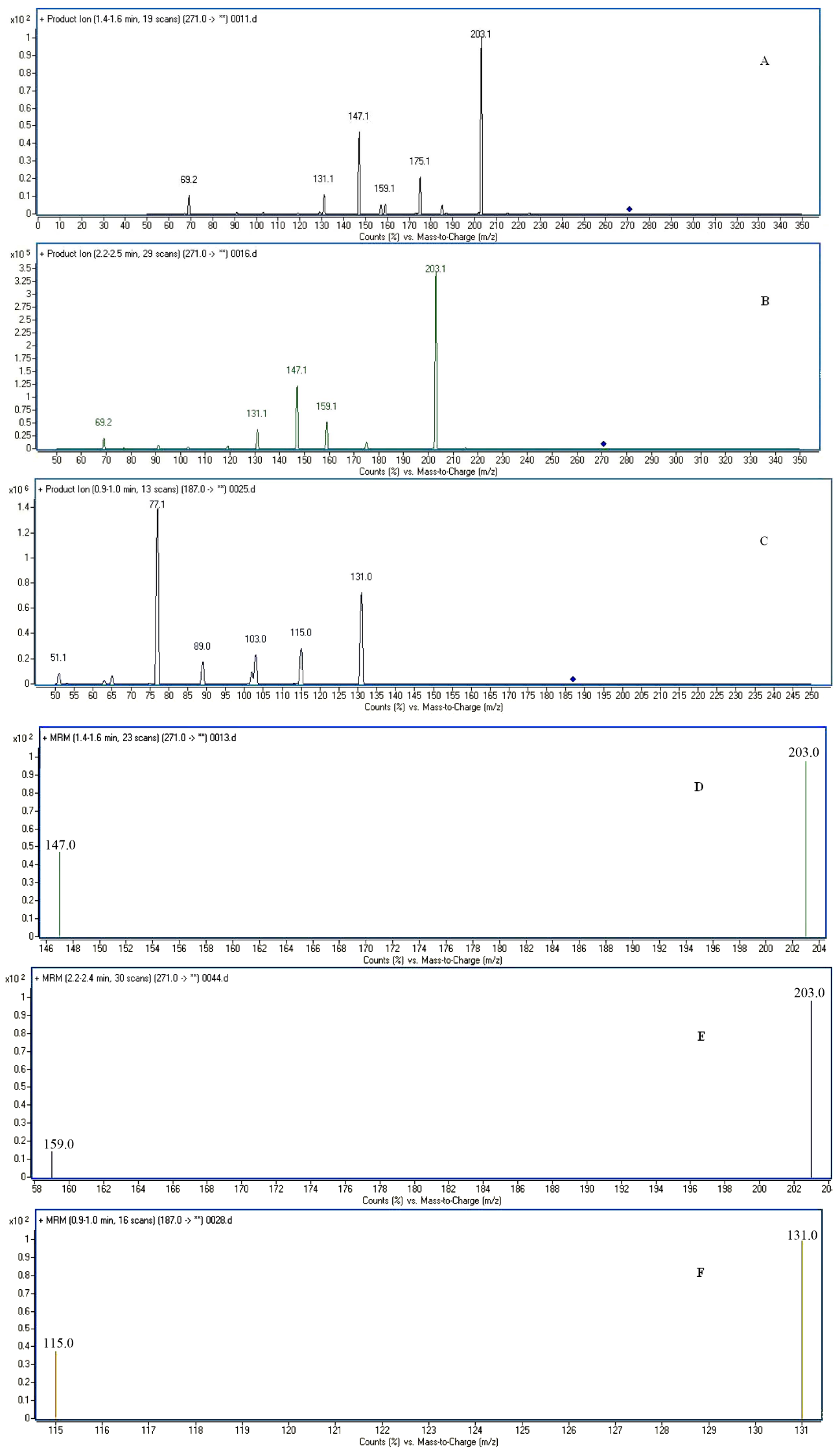


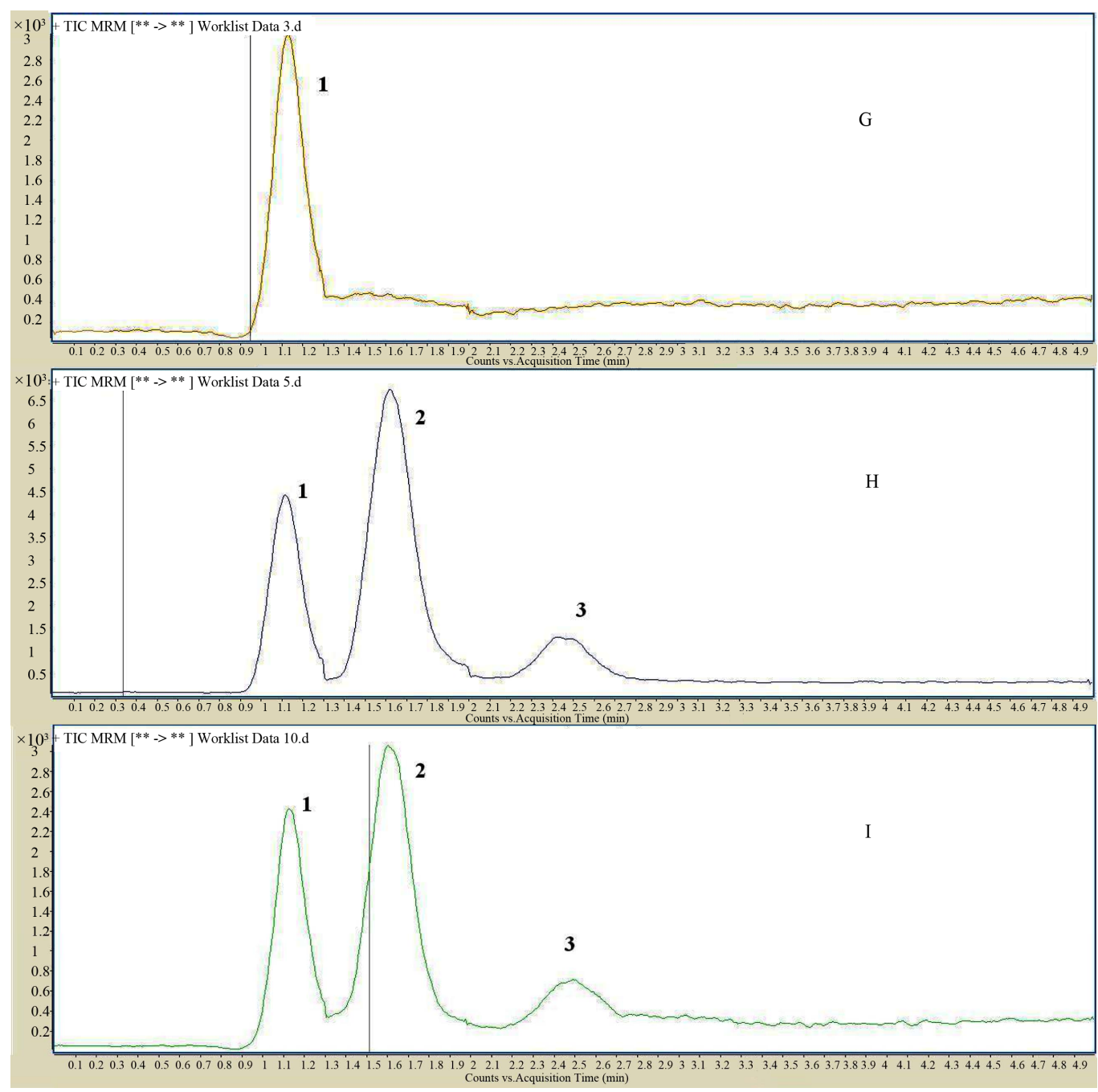

Figure 2. The MS/MS spectra of imperatorin (A); isoimperatorin (B); and I. S. psoralen (C); MRM spectra of imperatorin (D); isoimperatorin (E); and I. S. psoralen (F); MS-TIC chromatograms of blank plasma spiked with I. S. psoralen (G); MS-TIC chromatogram of blank plasma with reference substances and I. S. (H); and MS-TIC chromatogram of rat plasma samples collected at $60 \mathrm{~min}$ after intragastric administration (I); imperatorin (1), isoimperatorin (2), I. S. psoralen (3).

Table 1. Standard curves of the two coumarins from Angelicae dahuricae radix in rats' plasma.

\begin{tabular}{cccc}
\hline Compound & Concentration ranges $(\mathrm{ng} / \mathrm{mL})$ & Regression equation & Correlation coefficient \\
\hline imperatorin & $2.5-1000$ & $Y=1.0074 X+0.0115$ & 0.9997 \\
isoimperatorin & $2.5-1000$ & $Y=1.0663 X+0.0123$ & 0.9997 \\
\hline
\end{tabular}

\subsection{Accuracy and Precision of the Detection Method}

In general, the assay precision and accuracy were derived from the QC samples. From these data, the mean standard derivation and coefficient of variation at each level were determined. The intra-assay accuracy and precision in plasma for imperatorin and isoimperatorin ranged from $91.1 \%$ to $111.2 \%$ and $2.6 \%$ to $10.1 \%$, respectively, while inter-assay accuracy and precision for imperatorin and isoimperatorin ranged from $93.1 \%$ to $114.0 \%$ and
$3.3 \%$ to $10.9 \%$, respectively (Table 2 ).

\subsection{Stability}

Preliminary studies showed that imperatorin and isoimperatorin were stable under the conditions used in this work. The stability of processed samples was determined at $4^{\circ} \mathrm{C}$ in the autosampler for $24 \mathrm{~h}$. Imperatorin, and isoimperatorin did not degrade at these conditions, showing $100.5 \%$ and $99.1 \%$ recoveries, respectively. 
Table 2. Intra- and inter-assay precision of the determination of the two coumarins from Angelicae dahuricae radix in rats' plasma.

\begin{tabular}{|c|c|c|c|c|c|c|}
\hline \multirow[t]{2}{*}{ Spiked (ng/mL) } & \multirow{2}{*}{$\begin{array}{c}\text { Intra-assay precision }(\boldsymbol{n}=\mathbf{4}) \\
\text { Measured }(\mathrm{ng} / \mathrm{mL})\end{array}$} & \multicolumn{5}{|c|}{ Inter-assay precision $(n=4)$} \\
\hline & & R.S.D. $(\%)$ & Accuracy (\%) & Measured (ng/mL) & R.S.D. (\%) & Accuracy $(\%)$ \\
\hline \multicolumn{7}{|l|}{ imperatorin } \\
\hline 5 & $5.26 \pm 0.22$ & 8.4 & 105.2 & $5.42 \pm 0.16$ & 5.9 & 108.4 \\
\hline 25 & $26.83 \pm 1.48$ & 5.5 & 107.3 & $23.27 \pm 1.39$ & 6.0 & 93.1 \\
\hline 100 & $96.82 \pm 2.95$ & 3.0 & 96.8 & $93.72 \pm 3.28$ & 3.5 & 93.7 \\
\hline 250 & $261.50 \pm 8.63$ & 3.3 & 104.6 & $269.02 \pm 11.02$ & 4.1 & 107.6 \\
\hline 750 & $792.13 \pm 20.61$ & 2.6 & 105.6 & $774.36 \pm 25.55$ & 3.3 & 103.2 \\
\hline \multicolumn{7}{|l|}{ isoimperatorin } \\
\hline 5 & $5.56 \pm 0.28$ & 10.1 & 111.2 & $5.70 \pm 0.31$ & 10.9 & 114.0 \\
\hline 25 & $27.02 \pm 1.94$ & 7.2 & 108.1 & $23.47 \pm 1.96$ & 8.4 & 93.9 \\
\hline 100 & $92.22 \pm 3.96$ & 4.3 & 92.2 & $94.16 \pm 4.90$ & 5.2 & 94.2 \\
\hline 250 & $227.65 \pm 11.61$ & 5.1 & 91.1 & $241.95 \pm 14.32$ & 5.9 & 96.8 \\
\hline 750 & $800.22 \pm 30.41$ & 3.8 & 106.7 & $789.48 \pm 37.10$ & 4.7 & 105.3 \\
\hline
\end{tabular}

Table 3. Pharmacokinetic parameters of imperatorin in rats after administration of Radix Angelicae dahuricae extract $(n=8)$.

\begin{tabular}{|c|c|c|}
\hline Parameter & Imperatorin & Isoimperatorin \\
\hline $\mathrm{C} \max (\mathrm{ng} / \mathrm{mL})$ & $693.54 \pm 0.122$ & $336.31 \pm 0.208$ \\
\hline $\mathrm{T} \max (\mathrm{h})$ & $3.0 \pm 0.5$ & $3.0 \pm 0.5$ \\
\hline T 1/2 (h) & $5.0 \pm 0.2$ & $4.5 \pm 0.2$ \\
\hline $\operatorname{Ke}(\mathrm{h}-1)$ & $0.1386 \pm 0.014$ & $0.1406 \pm 0.068$ \\
\hline $\operatorname{AUC0}-\infty(\mathrm{ng} \mathrm{h} / \mathrm{mL})$ & $4750.2 \pm 2020.5$ & $3224 \pm 1415.3$ \\
\hline
\end{tabular}

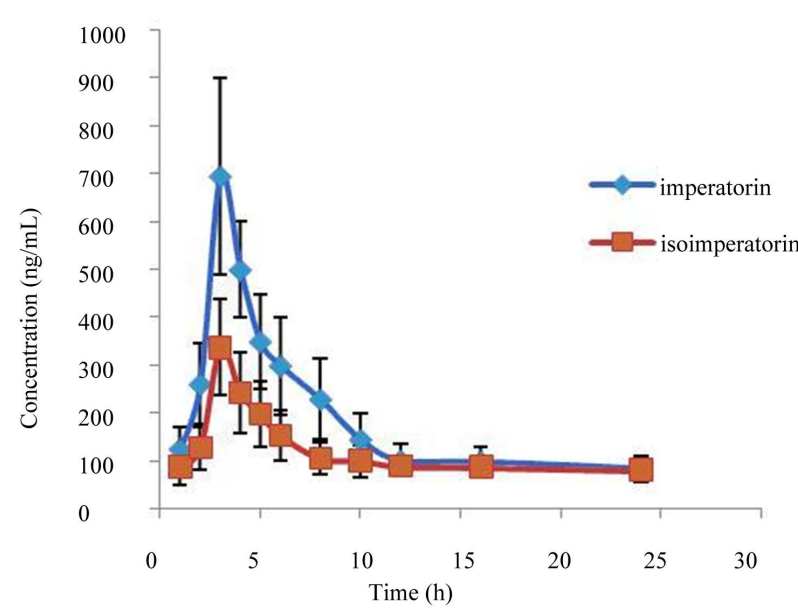

Figure 3. Means plasma concentration-time profiles of imperatorin and isoimperatorin after oral administration of Angelicae Dahuricae Radix extract.

\subsection{Applicability of the Analytical Method}

The method with $3 \mathrm{ng} / \mathrm{mL}$ LLOQ and $1 \mathrm{ng} / \mathrm{mL}$ LOD for the two coumarins is more sensitive than previously reported ones (LLOQ $=0.04 \mu \mathrm{g} / \mathrm{mL}$ ) [15]. So our method is more sensitive, and it can be used to study the pharmacokinetics, pharmacy and toxicity of Angelicae dahuricae radix. The results indicated that the analytical method is suitable to measure plasma concentrations of the compounds in pre-clinical studies.

The LC-MS/MS method developed was used to investigate the pharmacokinetics of imperatorin and isoimperatorin after an intragastric administration of a single dose to Wistar rats. The peak plasma level ( $C$ max) and the time to reach the peak plasma concentration ( $T$ $\max$ ) were obtained directly from the concentration-time data. The apparent elimination half-life $(T 1 / 2)$ was calculated as $0.693 / \mathrm{Ke}$. The area under the plasma concentration-time curve (AUC) was calculated by the trapezoidal rule. Each value is expressed as mean \pm S.D.

Table 3. shows the main pharmacokinetic parameters of imperatorin and isoimperatorin and Figure 3. shows the mean plasma concentration-time profiles of imperatorin and isoimperatorin after intragastric administration of imperatorin and isoimperatorin to eight rats.

From the mean plasma concentration-time profiles of imperatorin and isoimperatorin we can see that imperatorin and isoimperatorin were absorbed rapidly. The value of $T$ max of imperatorin is nearly the same as that of isoimperatorin, but $C \max$ of the two substances showed significant difference. T 1/2 indicated that the 
two components metabolized slowly. We surmised that quick absorption and distribution mainly resulted from the small molecular structures, and slow metabolism from their lipophilic properties.

\section{Conclusion}

As demonstrated in this assay, our method is sensitive, accurate and specific. In practical applications, this method meets the request of the present pharmacokinetic comparison and is suitable for the analysis of samples of Angelicae Dahuricae Radix.

\section{Acknowledgements}

The research was supported by "11th 5-year Medicine Health Scientific Foundation Program" (06MA376). Authors would like to thank Wang Guo-jia, Liu Jun, and Wang Wen-jun (Institute for Drug and Instrument Control Health Dept. GLD of PLA) for their collaboration in this study.

\section{REFERENCES}

[1] China Pharmacopoeia Committee, "Section One," In: China Pharmacopoeia Committee, Ed., Chinese Pharmacopoeia, China Medical Science Press, Beijing, 2010, pp. 97-98.

[2] J. Jin, C. S. Jin and D. L. Wu, "Historical Evolution of the Processing and Progress in Modern Research of Angelica dahurica," Anhui Medical and Pharmaceutical Journal, Vol. 16, No. 1, 2012, pp. 1-4.

[3] Y. C. Wang, C. H. Guo and X. L. Chen, "Observation of the Curative Effect of Xuefu Zhuyu Decoction with Baizhi and Huangqi on Headache after Stroke," Modern Journal of Integrated Traditional Chinese and Western Medicine, Vol. 18, No. 22, 2009, pp. 2628-2629.

[4] R. Hu, Z. L. Li and L. Yang, "Study on the Analgesic Effect of Imperatorin from Radix Angelicae Dahurica," Journal of Sichuan of Traditional Chinese Medicine, Vol. 30, No. 4, 2012, pp. 58-61. doi:10.1186/1479-5876-10-S2-A58

[5] X. L. Piao, H. Park and S. H. Baek, "Report the Isolation and the Antioxidative Activity against 2,2-Azobis (2Aminodinopropane) Dihydrochloride (AAPH)-Induced Cellular Damage of 11 Furanocoumarins from Angelica dahuricae," Journal of Ethnopharmacology, Vol. 93, No. 2-3, 2004, pp. 243-246. doi:10.1016/j.jep.2004.03.054

[6] F. Wang and C. Wang, "Study on the Relationship between the Anti-Aging Function and Antioxidation of Ethanol Extract from Angelicoe dahuricae Radix," China Pharmacy, Vol. 23, No. 7, 2012, pp. 599-602.

[7] W. H. Zhao, Y. X. Cao and J. Liu, "Research on Chronic Toxicology of Compound Radix Angelicae dahuricae Capsule," Journal of Shanxi Medical University, Vol. 37, No. 2, 2006, pp.160-163.

[8] R. Hu, Z. L. Li and L. Yang, "Anti-Inflammatory Effect of Imperatorin from Radix Angelicae dahurica on Rats with Acute Pleurisy," Journal of Emergency in Traditional Chinese Medicine, Vol. 21, No. 4, 2012, pp. 576579.

[9] H. Zhang, G. F. Hai and C. Zhang, "Experimental Studies on Analgesia and Anti-Febrile Effects of the Different Extracts from Radix Angelicae dahuricae," Journal of Xinxiang Medical College, Vol. 28, No. 4, 2011, pp. 431434.

[10] M. Y. Wang, M. Jia and Y. Y. Ma, "Pharmacological Effect of the Total Coumarins in Radix Amgelicae dahuricae," Lishizhen Medicine and Materia Medica Research, Vol. 16, No. 10, 2005, pp. 954-956.

[11] M. Y. Wang, M. Jia and Y. Y. Ma, "Pharmacological Effect of Four Linear Furocoumarins in Radix Angelicae dahuricae," Natural Product Research and Development, Vol. 22, No. 3, 2010, pp. 485-489.

[12] D. C. Wang, K. Li and X. Y. Xu, "Experimental Study Oil the Antipyretic-analgesic and Anti-inflammatory of Total Conmarins from Radix Angellcae dahurica," Chinese Journa1 of Information on TCM, Vol. 12 No. 11, 2005, pp. 36-38.

[13] K. Li, Q. W. Li and J. Liu, "Effect of AAD Anti-Tumor on Mice Bearing in U14 Cervical Cancer," Journal of Yanshan University, Vol. 36, No. 1, 2012, pp. 89-94.

[14] X. H. Qin, Z. L. Li and C. F. Zhu, "Study on the Pharmacodynamics of Total Coumarin of Radix Angelicae dahuricae in Treating Migrain," Joumal of Sichuan of Traditional Chinese Medicine, Vol. 26, No. 6, 2008, pp. 4-6.

[15] Y. B. Li, "Determination of Imperatorin in Rat Plasma by HPLC," Journal of Pharmaceutical and Biomedical Analysis, Vol. 40, No. 5, 2006, pp. 1253-1256. doi:10.1016/j.jpba.2005.09.030

[16] K. Ishihara, M. Fukutakeb and T. Asanob, "Simultaneous Determination of Byak-Angelicin and Oxypeucedanin Hydrate in Rat Plasma by Column-Switching High-Performance Liquid Chromatography with Ultraviolet Detection," Journal of Chromatography B: Biomedical Sciences and Applications, Vol. 753, No. 2, 2001, pp. 309-314. doi:10.1016/S0378-4347(00)00569-7

[17] Y. Xie, Y. Chen and M. Lin, "Developed and Validated for the Determination and Pharmacokinetic Study of Oxypeucedanin Hydrate and Byak-Angelicin after Oral Administration of Angelica dahurica Extracts in Mongrel Dog Plasma by HPLC," Journal of Pharmaceutical and Biomedical Analysis, Vol. 44, No. 1, 2007, pp. 166-172. doi:10.1016/j.jpba.2007.02.002

[18] Z. Han, Y. L. Zheng and L. J. Luan, "Analysis of Ochratoxin A and Ochratoxin B in Traditional Chinese Medicines by Ultra-High-Performance Liquid Chromatography-Tandem Mass Spectrometry Using $\left[{ }^{13} \mathrm{C}_{20}\right]$-Ochratoxin A as an Internal Standard," Journal of Chromatography A, Vol. 1217, No. 26, 2010, pp. 4365-4374. doi:10.1016/j.chroma.2010.04.052

[19] W. Yang, M. Ye and M. Liu, "Characterization of Coumarins in Radix Glehniae by Liquid Chromatography Coupled with Triple Quadrupole-Linear Ion Trap Mass Spectrometry," Journal of Chromatography A, Vol. 1217, 
No. 26, 2010, pp. 4587-4600.

doi:10.1016/j.chroma.2010.04.076

[20] S. C. Wang, Q. H. Chen and L. C. He, "Development and Validation of a Gas Chromatography-Mass Spectrometrymethod for the Determination of Isoimperatorin in Rat Plasma and Tissue: Application to the Pharmacokinetic and Tissue Distribution Study," Journal of Chromatography B, Vol. 852, No. 1-2, 2007, pp. 473-478.

[21] L. Ling, Z. L. Li and Y. X. He, "GC-MS Analysis of Extraction Products from Angelica dahurica (Fisch. ex Hoffm.) Benth. et Hook," Lishizhen Medicine and Materia Medica Research, Vol. 20, No. 2, 2009, pp. 306-307.

[22] L. Q. Zhu and H. G. Sheng, "GC-MS Analysis on Volatile Components of Radix Angelica dahuricate," Guangzhou Chemical Industry, Vol. 40, No. 23, 2012, pp. 103105.

[23] A. H. Zhao, X. W. Yang and X. B. Yang, "GC-MS Analysis of Essential Oil from Root of Angelica dahurica cv. Qibaizhi," China Journal of Chinese Materia Medica, Vol. 36, No. 5, 2011, pp. 603-607.

[24] Y. C, Lee, C. Y. Huang and K. C. Wen, "Determination of Liquiritin, Glycyrrhizin, Hesperidin, Cinnamic Acid, Cinnamaldehyde, Honokiol and Magnolol in the Traditional Chinese Medicinal Preparation Wu-Ji-San by HighPerformance Liquid Chromatography," Journal of Chromatography A, Vol. 692, No. 1-2, 1995, pp. 137-145. doi:10.1016/0021-9673(94)00740-Z

[25] X. G. Zheng, X. W. Zhang and X. N. Sheng, "Simultaneous Characterization and Quantitation of 11 Coumarins in Radix Angelicae dahuricae by HPLC-ESI-MS/ MS," Journal of Pharmaceutical and Biomedical Analysis, Vol. 51, No. 3, 2010, pp. 599-605. doi:10.1016/j.jpba.2009.09.030

[26] C. Y. Zhang, Z. G. Li and Q. X. Wang, "Study on HPLC Fingerprint of Processed Radix Angelicae dahuricae,"
Journal of Chinese Medicinal Materials, Vol. 30, No. 11, 2007, pp. 1374-1378.

[27] T. T. Wang, X. H. Chen and Q. Q. Hu, "RP-HPLC Fingerprint for Quality Assessment of Radix Angelicae dahuricae," Acta Pharmaceutica Sinica, Vol. 41, No. 8, 2006, pp. 747-751.

[28] J. Zhang, Y. S. Wang and X. R. He, "Studies on Identification of Radix Angelicae dahuricae by HPLC Fingerprint," Chinese Journal of Experimental Traditional Medical Formulae, Vo1, 13, No. 7, 2007, pp. 1-4.

[29] G. H. Cui, L. Q. Huang and X. Li, "A New Molecular Identification Method: Anchored Primer Amplification Polymorphism DNA," Acta Pharmaceutica Sinica, Vo1. 42, No. 3, 2007, pp. 329-335.

[30] J. Y. Deng, G. H. Gao and C. J. Zhao, "Determination of the Contents of Two Coumarins in Angelica dahurica from Different Regions by HPLC," Journal of Shenyang Pharmaceutical University, Vol. 21, No. 5, 2004, pp. 354-357.

[31] Z. Q. Mao, X. L. Meng and Y. L. Li, "Determination of Xanthotoxol, Bergapten, Imperatorin and Isoimperatorin in Angelicae dahuricae Radix by HPLC," China Pharmacist, Vol. 14, No. 2, 2011, pp. 205-207.

[32] Y. Y. Zhao, H. Sun and M. Wang, "Simultaneous Quantitative Determination of Five Coumarins in Angelicae dahuricae Introduced in Jiangsu by HPLC," Chinese Wild Plant Resources, Vol. 32 No. 1, 2013, pp. 56-59.

[33] R. Deng, J. Zhang and W. Z. Luo, "Quantitative Determination of Six Coumarins in Angelica dahurica var. formosana by RRLC-UV," China Journal of Chinese Materia Medica, Vol. 35, No. 23, 2010, pp. 3184-3187.

[34] Y. Y. Ma, S. H. Zhong and M. R. Jia, "Determination of Total Content of Coumarin in Angelica dahurica by UV Spectrophotometry," West China Journal of Pharmaceutical Science, Vol. 20, No. 2, 2005, pp. 159-160. 